Cinémas

Revue d'études cinématographiques

Journal of Film Studies

\title{
L’image du cinéma dans les romans canadiens-français de 1896 à 1930
}

\section{Micheline Tremblay}

Volume 6, numéro 1, automne 1995

Le cinéma muet au Québec et au Canada : nouveaux regards sur une pratique culturelle

URI : https://id.erudit.org/iderudit/1000963ar

DOI : https://doi.org/10.7202/1000963ar

Aller au sommaire du numéro

Éditeur(s)

Cinémas

ISSN

1181-6945 (imprimé)

1705-6500 (numérique)

Découvrir la revue

Citer cet article

Tremblay, M. (1995). L'image du cinéma dans les romans canadiens-français de 1896 à 1930. Cinémas, 6(1), 133-148. https://doi.org/10.7202/1000963ar
Résumé de l'article

L'auteure retrace ici la place qu'occupe le cinéma dans la littérature et relève les jugements dont il fait l'objet. Dès son arrivée au Canada français, le cinéma attire les foules. Pourtant, les oeuvres romanesques témoignent peu de cet engouement pour le cinéma. Rares sont les romanciers dont les personnages fréquentent les salles obscures. C'est que le cinéma apparaît en relation avec tout ce qui s'oppose à l'idéologie dominante : c'est un phénomène urbain, américain, et qui propage des valeurs immorales aux yeux des groupes dominants des sociétés civile et politique. Point étonnant, donc, qu’un lourd discrédit social pèse contre le cinéma. 


\title{
L'image du cinéma dans les romans canadiens-français de 1896 à 1930
}

\author{
Micheline Tremblay
}

\section{RÉSUMÉ}

Lauteure retrace ici la place qu'occupe le cinéma dans la littérature et relève les jugements dont il fait l'objet. Dès son arrivée au Canada français, le cinéma attire les foules. Pourtant, les œuvres romanesques témoignent peu de cet engouement pour le cinéma. Rares sont les romanciers dont les personnages fréquentent les salles obscures. C'est que le cinéma apparaît en relation avec tout ce qui s'oppose à l'idéologie dominante: c'est un phénomène urbain, américain, et qui propage des valeurs immorales aux yeux des groupes dominants des sociétés civile et politique. Point étonnant, donc, qu'un lourd discrédit social pèse contre le cinéma.

\section{ABSTRACT}

The author here outlines the place that cinema has held in literature and summarizes the judgements literature has expressed about it. Right from the beginning, movies attracted crowds in French Canada, but novels of the period offer little testimony to popular enthusiasm for the cinema. Few novelists presented characters who frequented the darkened halls. Cinema appeared in close association with all the things to which the dominant ideology stood opposed : it was an urban phenomenon, an American phenomenon, and one which, in the view of the dominant groups in civil and political society, promoted immoral values. Its success came therefore despite the heavy load of social opprobrium that weighed against it. 
Faut-il rappeler que la première représentation publique du Cinématographe a eu lieu le 28 décembre 1895 à Paris et qu'à peine six mois plus tard, en juin 1896, les Montréalais pouvaient également assister à un spectacle de "vues animées '". Curieusement, dans cette société où l'on prône l'idéologie de conservation, où le changement est proscrit, où l'on se méfie de tout ce qui est étranger (particulièrement en provenance des États-Unis), le cinéma se répand comme une traînée de poudre $^{2}$. Et ce, malgré les nombreuses luttes du clergé!

Constatant le succès du cinéma, nous avons voulu cerner la vision que donnent les romanciers de l'entrée du cinéma au Canada français ${ }^{3}$. Après un bref survol quantitatif permettant de jeter un regard global sur l'importance de la présence du cinéma dans les romans, nous nous attarderons, d'un point de vue plus qualitatif, à examiner les différentes manifestations de cette présence. Plus particulièrement, nous tenterons de cerner comment les romanciers présentent les relations entre leurs personnages et le cinéma et l'impact de ce nouvel art sur la société. Les romans choisis s'échelonnent de 1896 - date de l'arrivée du cinéma au Canada - à 1930, date qui coïncide avec le développement du cinéma parlant. Afin de bien circonscrire les modalités de la pénétration du cinéma dans le roman, tous les romans des deux premières décennies ont été retenus, soit 64 romans. Puis, une fois la présence du cinéma suffisamment attestée et régulière, nous avons choisi, pour la décennie suivante, un échantillon ${ }^{4}$ composé de $40 \%$ de l'ensemble des romans publiés, soit 40 romans. Au total, cette recherche s'appuie donc sur une solide base documentaire composée de plus de 100 romans.

Dans ces œuvres, nous avons relevé toutes les références explicites au cinéma ${ }^{5}$ et elles seules ont servi de base à notre analyse. Cette démarche rigoureuse peut paraître contraignante et limitative. Il est vrai qu'elle nous a parfois obligée à abandonner des passages fort intéressants dans lesquels, intuitivement, on sentait la présence cinématographique. Par contre, elle nous a permis d'éviter les écueils des interprétations trop subjectives ${ }^{6}$. 


\section{Analyse quantitative}

Au cours de ses 30 premières années d'existence, le cinéma occupe peu de place dans les romans canadiens-français. Vingtcinq seulement des 104 romans à l'étude en font mention dont 2 sont écrits par des femmes. Vingt et un personnages ${ }^{7}$ seulement le fréquentent et quelques-uns à peine sont décrits à l'intérieur même d'une "salle obscure". De ces 21 personnages, 15 sont masculins et 6 sont féminins ${ }^{8}$. Ce sont tous des jeunes et des adultes; aucun enfant ne le fréquente. Autant dans le cas des romanciers que des personnages, les chiffres laissent penser que le cinéma, du moins à cette période, est un phénomène presque exclusivement masculin; cela va donc à l'encontre de l'idée voulant qu'à ses débuts, le cinéma ait surtout attiré des femmes ${ }^{9}$. Si aucun réalisateur ni aucun titre de films n'y sont mentionnés, quatre acteurs y sont cependant nommés, tous américains (Charlie Chaplin, Pearl White, Mary Pickford et Douglas Fairbanks), montrant déjà la nette prédominance de ce cinéma. Ces quelques éléments quantitatifs montrent à l'évidence que le cinéma pénètre lentement dans le roman et ce, contrairement à ce qui se passe dans la réalité.

\section{Analyse qualitative}

Ce n'est qu'en 1903 qu'apparaît la première référence cinématographique dans un roman canadien-français, suivie d'une autre quelque cinq ans plus tard ${ }^{10}$. Allusions brèves, discrètes et timides où les romanciers ne font qu'utiliser un élément de la technique cinématographique comme élément de comparaison. Pourtant, en 1912, dans L'Envers du journalisme, Alfred Mousseau reconnaît implicitement que le cinéma attire les foules en décrivant une curieuse projection où il s'inspire d'un fait réel :

Ils furent ébahis du spectacle formidable qui s'offrit à eux : dix mille spectateurs acclamaient avec frénésie la victoire d'un homme politique jouissant d'une grande popularité. Les employés chargés de faire les projections durent faire passer son portrait plusieurs fois sur la toile. À chaque fois, les acclamations montaient (Mousseau, 1912, p. 156). 
Bien que Mousseau ne fasse pas clairement allusion au cinéma, le lien avec l'événement organisé par Ernest Ouimet " semble assez évident. C'est toutefois Hector Bernier dans Ce que disait la flamme (1913) qui reconnaîtra, pour la première fois dans le roman canadien-français, le cinéma comme un phénomène urbain et de masse. Non seulement y décrit-il l'ampleur de l'engouement pour le cinéma, mais il le situe dans une problématique importante: celle de l'opposition ville / campagne. En effet, le héros, Paul Garneau, affirme n'être heureux qu'à la campagne:

Deux ou trois jours de griserie, de poignées de main qui réchauffent, de sourires qui font du bien, de vieille routine, de vues animées, de promenades, de gazoline... Me voilà rassasié, déjà triste... Il me faut l'espace, la montagne, les arômes de la forêt, les lacs, tu sais, le matin, quand tout recommence à vivre... (Bernier, 1913, p. 83)

Mais en cela, il s'oppose à toute la jeunesse, à tous ses amis qui, parce qu'il " $[\ldots]$ ne se rend jamais aux spectacles des vues animées [le considèrent comme] un imbécile. Ne pas faire comme eux et l'asile, c'est presque la même chose!» (p. 85-86). Bernier met bien en lumière la grande popularité du cinéma auprès des jeunes; ne pas aimer le cinéma, c'est se situer en dehors des normes tacites de la jeunesse de l'époque. La pression sociale des pairs pèse lourdement sur celui qui tente d'échapper à l'attraction des petites vues. Par rapport au discours officiel prônant le retour à la terre, Paul Garneau n'est nullement un marginal; par contre, considéré du point de vue de la jeunesse des années 1910-1920, c'est un rétrograde. Ce roman met ainsi nettement en évidence l'écart entre les valeurs véhiculées idéologiquement par la société et celles réellement vécues dans le quotidien. Notons également que Bernier, en partageant les points de vue de son narrateur et de son personnage, incite ainsi le lecteur à se rallier à cette opinion dévalorisante de la ville et, par conséquent, du cinéma.

Le roman le plus connu de cette période est sans doute celui de Louis Hémon, Maria Chapdelaine (1914). Phénomène urbain, le cinéma est également présenté ici comme un phéno- 
mène américain. Voulant convaincre Maria de l'épouser, Lorenzo Surprenant lui fait miroiter les avantages de la ville américaine et de ses cinémas où on peut entrer " [...] pour un nickel, cinq cents, et rester deux heures à pleurer et à rire. Oh! Maria! Penser que vous ne savez même pas ce que c'est que les vues animées!" (Hémon, 1970, p. 155). Mais comment lui expliquer

[...] le spectacle prodigieux des cinématographes [...] et en raconter les péripéties ordinaires: l'histoire touchante des petites filles abandonnées ou perdues dont la vie est condensée sur l'écran en douze minutes de misère atroce et trois minutes de réparation et d'apothéose dans un salon d'un luxe exagéré... Les galopades effrénées des cow-boys à la poursuite des Indiens ravisseurs; l'épouvantable fusillade; la délivrance ultime des captifs, à la dernière seconde, par les soldats qui arrivent en trombe, brandissant magnifiquement la bannière étoilée...[...] Après une minute d'hésitation, il secoua la tête reconnaissant son impuissance à peindre toutes ces choses avec des mots (Hémon, 1970, p. 154155).

Avec cette œuvre de Hémon, le cinéma fait un pas de plus dans l'univers romanesque puisqu'une description littéraire s'attarde au contenu, à la durée et à la structure des films. Déjà, se dégagent certaines caractéristiques des films américains. En effet, au niveau thématique, on reconnaît les mélodrames pathétiques, les westerns, le patriotisme américain alors qu'au niveau de la structure du récit, l'action et le suspens sont déjà en place, le happy end ne survenant que dans les toutes dernières secondes du film. Pour Lorenzo Surprenant, le cinéma est si extraordinaire qu'il n'arrive pas à le décrire avec des mots et il constate ainsi l'infériorité de la langue face à la richesse des images mouvantes.

Mais l'aspect le plus important de la contribution de Hémon se situe sur un autre plan: il assimile le cinéma aux États-Unis. En effet, Lorenzo Surprenant est exilé aux États-Unis et, par le fait même, considéré comme un double traître par la famille Chapdelaine; d'abord parce qu'il a quitté la terre, ensuite parce qu'il a quitté le pays. C'est ainsi que le premier personnage romanesque à admettre se plaire au cinéma est considéré comme 
un transfuge. En associant le cinéma non seulement à la ville, mais également aux États-Unis et à l'exilé, Louis Hémon lui confere un discrédit social déjà pressenti dans Ce que disait la flamme (1913) d'Hector Bernier et qui est en train de prendre place. Cela souligne aussi la remarquable compréhension qu'a Louis Hémon de la société canadienne-française.

Le premier personnage à être décrit à l'intérieur d'une salle de cinéma est Donat Mansot dans Le Membre (1916) de Damase Potvin. Ayant accepté des pots-de-vin importants, ce personnage sait que son poste de député est menacé et qu'il risque la prison; ne pouvant dormir, il se rend dans un "scope" où l'on présentait un drame policier qui l'intéressa beaucoup.

A la fin, il rit franchement de la mine piteuse et si naturelle que sut prendre l'acteur chargé du rôle de la victime quand $[\ldots]$ il dut $[\ldots]$ tomber dans les filets si habilement tendus du détective... (Potvin, 1916, p. 122)

Cette citation est doublement intéressante; d'une part, elle souligne l'aspect cathartique du cinéma et d'autre part, elle montre l'incapacité du personnage-spectateur à s'identifier au personnage filmique et ce, malgré le parallélisme évident entre les deux situations. Comme si, parce que projetée sur un écran, la situation devenait autre, distante de lui-même. Fiction et réalité demeurent étanches; Donat Mansot ne franchit pas la distance entre ce personnage et lui-même et il peut donc consommer le film sans aucun risque. Par contre, le narrateur, conscient de l'analogie entre les deux situations, ridiculise son personnage et crée ainsi une complicité avec le lecteur sur le dos de son personnage.

Au cours de la décennie suivante, le cinéma se propage rapidement et dans les romans, c'est essentiellement à Montréal qu'on le rencontre. Le soir, les rues de la métropole deviennent un véritable chaos mais après 11 heures du soir, tout ce mouvement se calme, car " [...] les théâtres et les cinémas déversent les dernières foules" (Berton, 1926, p. 127). Même les petites villes sont dotées de ces établissements. Résidente de Saint-Hyacinthe, Alberte Dumont dans L'Homme tombé (1924) de Harry Bernard 
fréquente "[...] régulièrement l'unique théâtre de la ville" (p. 94); elle déplore cependant le fait que la ville ne compte qu'un seul cinéma et que cela l'empêche de suivre toutes les primeurs cinématographiques.

Jeanne-Marie Clerc a remarqué, dans les romans français contemporains, que "[...] la forme la plus anodine de ce discrédit s'explique souvent par la relation fréquemment établie entre cinéma [...] et désœuvrement ou passivité» (p. 32). Au Canada français, le même constat peut être fait. Ce désœuvrement apparaît nettement, dans les années 20, chez plusieurs personnages dont, pour n'en mentionner que quelques-uns, Yves Marin dans L'Iris bleu (1923) de Jules-Ernest Larivière et Étienne Normand dans L'Homme tombé (1924) de Harry Bernard. Homme de la campagne, Yves Marin ne sait que faire pour passer un dimanche après-midi à la ville et il "[...] s'enhardit à entrer dans un cinéma" (Larivière, 1923, p. 36). Le verbe, connotant une certaine audace, rappelle tous les interdits pesant contre le cinéma du dimanche, spécialement à Montréal. Dans le second cas, lorsque Étienne Normand se retrouve seul à Montréal, ne sachant que faire, il " [...] entra au théâtre pour le reste de la soirée» (Bernard, 1924, p. 156).

Mais ce discrédit, on l'attribue surtout à l'influence néfaste du cinéma. Certains romanciers iront même jusqu'à montrer comment la fréquentation assidue du cinéma a des conséquences négatives sur la vie de leurs personnages. De plus en plus conscients de l'immense pouvoir du cinéma, surtout auprès des jeunes, les romanciers réagissent en l'attaquant férocement. Arsène Goyette et Henri Deyglun dénoncent avec virulence le cinéma, qui incite au crime et à la débauche. Dans L'Ineffaçable Souillure (1926) d'Arsène Goyette, le juge Madore se rend au pénitencier afin d'enquêter sur l'influence de l'éducation sur le crime; un officier lui affirme que des prisonniers lui " [...] ont confessé qu'en fréquentant les vues animées où l'immoralité coule à flots continus sur l'écran, ils avaient puisé l'idée première de leurs débauches exécrables [...]" (Goyette, 1926, p. 60).

Ce côté immoral du cinéma, Henri Deyglun le dénonce aussi dans Les Aventuriers de l'amour (1929). Il y fustige les films d'aventures américains dont il dénonce l'influence néfaste sur la 
jeunesse: "Toute la nouvelle génération nourrie spirituellement par les films en séries de nos voisins a un goût plus ou moins prononcé pour les aventures" (p. 20) et c'est ce goût de l'aventure qui l'incitera à commettre des crimes crapuleux comme celui des antihéros de ce roman. De même, Jules-Ernest Larivière discrédite le cinéma à cause de son contenu aux valeurs morales trop libertaires. Dans L'Associée silencieuse (1925), son héros songe à abandonner sa carrière de journaliste, car on lui a confié une tâche indigne de son talent, celle d'interviewer une actrice :

- Et quel déboire! Mon chef m'envoya interviewer une étoile de cinéma trois fois divorcée [...] écrire deux colonnes de louanges sur [...] une cabotine à la voix rauque, dont la myriade de badauds venait chaque jour admirer sur l'écran les charmes postiches (p. 5).

Le vocabulaire familier et méprisant s'ajoute au contenu dénigrant. Notons le caractère faux du cinéma qui se dégage de cette citation: fausseté du talent de l'actrice, fausseté de ses charmes. Plus loin, ce personnage s'insurgera contre "la presse jaune " qui, cherchant à répondre au désir de la clientèle, juxtapose «[...] la photo d'un saint évêque sur la même page que celle du dernier meurtrier qu'attend le bourreau ou encore $[\ldots]$ celle d'un brave curé de campagne voisinant celle d'une cabotine de cinéma trois ou quatre fois divorcée" (Larivière, 1925, p. 5). La symétrie de l'énumération, qui assimile le saint évêque au curé et le meurtrier à la cabotine de cinéma, en dit long sur la perception du cinéma!

Par ailleurs, le cinéma est censé susciter chez certaines femmes des rêves de richesse, de luxure, de vie axée sur le plaisir et la satisfaction des besoins qui dérogent fortement de l'idéal social de l'époque. Les lourdes obligations de la vie quotidienne - le devoir et les sacrifices - apparaissent bien ternes à certaines jeunes femmes, considérées comme superficielles, qui cherchent à vivre au-delà du réel, à transformer leur réalité en cinéma. Victime du cinéma, Alberte Dumont dans L'Homme tombé (1924) ruinera sa vie de couple tandis que Ruth Madore de L'Ineffaçable Souillure (1927) prendra conscience à temps, selon le romancier, 
de l'inanité de sa vie: "J'ai beau me repaitre de romans qui passionnent, je souffre et je pleure [...] C'est en vain que je fréquente le théâtre et les vues animées [...] Je sens dans mon cœur un vide immense..." (Goyette, p. 27-28).

Les romans, le théâtre et le cinéma constituent pour ce personnage des moyens de s'étourdir; le discrédit social n'atteint donc pas seulement le cinéma, mais tous les arts considérés comme arts de l'illusion, du "faux". Au Canada français, sous l'influence de l'idéologie cléricale, un être sain doit nourrir son intelligence et sa raison et non son imagination, cette "folle du logis». En somme, tout ce qui est de nature à exciter l'imaginaire est dénigré.

Si le cinéma a une influence aussi néfaste, c'est beaucoup parce qu'il est identifié aux États-Unis. Par le biais d'une attaque du cinéma, Jean-Charles Harvey dénonce dans son roman Marcel Faure (1922) la mentalité américaine:

— Ah! Les Américains! [...] Un tas d'abrutis! Une nation de païens où le mariage est un jeu et le divorce un sport. Les Américains! Des chercheurs de plaisir et d'excitations sensuelles, [...] Nous sommes aux antipodes. Eux, qu'adorent-ils, après tout? La chair, le métal, le cinéma (p. 179).

Condensées en quelques mots se retrouvent dans cette critique virulente les grandes accusations issues des préjugés cléricaux contre le cinéma américain: il propage une nouvelle mentalité où la recherche du plaisir — plaisir de la chair, plaisir de l'argent - devient le but de la vie. Jules-Ernest Larivière dans L'Iris bleu (1923) dénonce l'insignifiance des films américains et suggère le refuge à la campagne pour contrer leur influence grandissante :

Mon Dieu que c'était abrutissant. On y représentait un drame américain, vols, brigandages et autres pareilles infamies, le tout joué par des cabotins américains représentés dans un théâtre canadien. Si c'est avec de telles représentations que l'on compte lutter contre l'américanisme! Heureusement, nous sommes là, nous, les fils des campagnes, et nous saurons toujours y opposer un mur solide (p. 65-66). 
Jugement critique sévère sur le contenu des films que le romancier insère dans une avenue plus large: celle de l'américanisme. Devenu synonyme d'américanisation, le cinéma se trouve ainsi au confluent de deux courants de pensée correspondant à un net clivage social. D'une part, le clergé et l'élite canadienne-française qui, voulant protéger leur langue et leur foi et freiner le courant d'émigration vers le Sud, cherchent à amplifier la méfiance contre les États-Unis. D'autre part, le petit peuple, attiré pour ne pas dire fasciné par la modernité, par le travail dans des manufactures qui rapporte de l'argent, par les images de plaisir et de vie facile, qui n'hésite pas à fréquenter le cinéma, à s'imprégner de la culture et des valeurs américaines et, souvent même, à s'exiler aux États-Unis.

Puisque, socialement, le cinéma semble avoir si mauvaise réputation, on peut se demander ce qui attire les individus aux représentations cinématographiques. Si les curés semblent craindre la promiscuité des salles obscures, les romanciers n'y font pourtant aucune allusion avant la fin des années 30. Quelle est donc la nature de ce désir qui pousse les personnages au cinéma?

Dès 1924, dans L'Homme tombé, Harry Bernard répond à cette question. Son personnage, Étienne Normand, ne sachant que faire, entre sans enthousiasme dans un cinéma où "[...] comme la plupart des habitués, qui ont vu les mêmes dislocations cent fois [et qui] se plaignent de leur monotonie, [mais qui continuent] de les voir par besoin de regarder quelque chose" (p. 156). Malgré l'insignifiance du film, toute la salle continue à le visionner. Quelle est donc cette force qui les contraint à rester sur leur siège? "Besoin de regarder quelque chose", répond le romancier, sans nous renseigner pour autant sur la nature de cette pulsion voyeuriste. Pulsion qui peut s'expliquer, si l'on en croit Jean-Louis Baudry (1978), par ce qu'il appelle l'identification primaire, où le spectateur s'identifie moins avec le contenu qu'avec ce que met en scène le spectacle. Cette identification primaire peut se prolonger dans une identification secondaire, celle du spectateur au personnage filmique. Dans les romans de notre corpus, cette forme d'identification se retrouve pour la première fois dans le personnage d'Alberte Dumont dans L'Homme tombé (1924). 
Séduite par la richesse, les plaisirs, la vie facile que lui présente le cinéma, Alberte rêve de transformer sa vie, car le quotidien ne lui suffit plus. Fréquemment, elle se rend seule à Montréal pour assister aux primeurs cinématographiques; elle se fera même prescrire une cure de repos aux États-Unis où elle espère, en son for intérieur, avoir la chance de rencontrer ses acteurs favoris. De retour au Québec, elle incite son mari à quitter son travail pour déménager à Montréal afin de pouvoir plus facilement s'adonner à sa passion. Délaissant mari et enfant, le seul but de sa vie devient, comme dans les films américains dont elle se gave, le plaisir et la satisfaction de ses désirs. Présentée par le romancier comme un personnage faible, Alberte Dumont vit tellement en empathie avec les personnages de l'écran qu'elle voudrait, à la fin du film, réintégrer une réalité qui soit celle du cinéma.

Roman à thèse, le texte de Harry Bernard veut démontrer que l'engouement pour la ville et la passion du cinéma, en provoquant la désaffection des valeurs prônées par l'idéologie dominante, ne peuvent mener qu'à la détérioration du couple et à la déchéance des êtres ${ }^{12}$. On croyait, en effet, que le cinéma, en intoxiquant l'imagination de scènes irréelles, provoquait chez le spectateur un certain désenchantement face à sa vie quotidienne ${ }^{13}$. Résultat: le cinéma dégoûte du monde vrai. Le personnage de Harry Bernard illustre parfaitement cet impact immoral du film sur l'individu.

Contrairement à sa femme, Étienne Normand dans L'Homme tombé (1924) n'est pas un adepte du cinéma. Pourtant, l'univers cinématographique provoque en lui une profonde remise en question de ses valeurs comme s'il préférait l'image des autres à la sienne. La morale présentée par les personnages filmiques est aux antipodes du jansénisme qui est le sien. Étienne Normand dédaigne les biens matériels et préconise le sacrifice, le don de soi. Mais les personnages de l'écran, eux,

[...] ne se souciaient pas de réformer le monde, de mériter aux yeux de l'humanité. [...] Ils prenaient à l'existence ce qu'elle a de meilleur, de plus tangible et de plus savoureux. N'étaient-ils pas les plus sages? Cette conception des choses ne valait-elle pas toutes les philosophies (Bernard, 1924, p. 159)? 
Sous l'influence de sa femme, Étienne Normand subit la "tentation du plaisir" et voilà la raison d'ailleurs pour laquelle le romancier intitule son œuvre L'Homme tombé. Tombé parce que, entre autres, l'image qu'il a de soi ne résiste pas à l'image des autres que lui propose le cinéma: cela est significatif de la puissance du cinéma qui peut remettre en question l'image qu'on a de soi. Même si le romancier considère Étienne Normand - et le titre en est la preuve - comme un être faible, en faisant partager son point de vue au lecteur, Harry Bernard amène celui-ci à envisager pour lui-même cette remise en question. Par le fait même, il amplifie le poids à donner à cette remise en question des valeurs morales et sociales.

Malgré leurs profondes différences, Alberte Dumont et Étienne Normand sont subjugués par les stars. Cette admiration se rencontre également chez d'autres personnages de romans et elle se nourrit par l'achat de revues qui accordent une très large place à l'iconographie au détriment du texte qui n'y est plus, bien souvent, qu'un bref commentaire à la photographie. Ces revues, généralement, ne manifestent aucune approche critique: "C'est le domaine où triomphent l'effusion délibérément aveuglée, l'adhésion totale et mystifiée, le discours de l'amour" (Aumont et al., p. 4). Cet intérêt pour les revues de cinéma, on en trouve trace dès 1918 dans Les Aventures extraordinaires de deux Canayens de Jules Jéhin. Le roi de Prusse reçoit un message au moment où, allongé, il «[...] feuilletait un magazine de théâtres et de cinémas dans lequel il admirait les portraits de jolies actrices et se plaisait à considérer leurs formes séduisantes [...]" (p. 55). Des magazines où les photographies suggestives des vedettes provoquent le désir de l'homme. Dans les romans antérieurs, les seuls personnages féminins retenus étaient des "petites filles"; voici maintenant apparaître la femme dans un halo de formes sensuelles. Malgré la discrétion de cette description, on peut s'interroger sur l'impact de ce plaisir pris à admirer ces "formes séduisantes" à une époque dominée par le jansénisme et où tout ce qui avait un rapport avec la "chair » était péché! C'est ensuite dans un roman de Harry Bernard, La Maison vide (1926), que l'on revoit cet engouement pour les revues: $\mathrm{M}^{\mathrm{me}}$ Dumontier reçoit de nombreuses revues de Paris et de New York qui 
[...] publiaient par douzaines les photos de princesses et d'actrices, qui lançaient à tour de rôle, sans distinction d'âge ni de situation, une mode excentrique ou un engouement pour tels exercices physiques. Madame Dumontier avait lu aussi, sans les comprendre, les romans sportifs de Montherlant, poèmes en prose à la gloire du muscle (p. 168-169).

L'ironie de la dernière partie de cette citation en dit long sur la considération que le romancier porte à son personnage. De plus, en alliant la "princesse" à "l'actrice", Bernard nous amène à réfléchir sur les possibles ressemblances entre la monarchie et le star system. Comme le prétend Edgar Morin: " [...] la star et le roi sont des êtres de chair contaminés par leur rôle" (p. 114). Alberte Dumont s'extasie devant Douglas Fairbanks, un acteur "[...] connu du monde entier" (Bernard, 1924, p. 58) et songe même à un voyage aux États-Unis à cause de "[...] l'attirance qu'exerçaient [...] les acteurs de cinéma [...]” (p. 94-95). Soulignons toutefois, en terminant, que ce phénomène est encore peu fréquent dans les romans de cette période.

Le cinéma cumule tout ce qui est honni par l'idéologie de conservation. C'est un phénomène urbain dans une société prônant le retour à la terre, un phénomène massivement américain dans une société où l'on craint l'influence des États-Unis et il représente le pouvoir de la "chair" dans une société prude et janséniste. Il va sans dire qu'il va à l'encontre des valeurs sociales canadiennes-françaises. C'est sans doute la raison pour laquelle, au cours des premières décennies de l'existence du cinéma, les romanciers canadiens-français lui réservent un accueil discret et prudent. Discret par le nombre de romans et de personnages qui en font mention et par le peu d'importance des occurrences; prudent parce qu'ils n'osent le présenter positivement. La plupart des romanciers dépeignent le cinéma comme un élément de corruption endossant en cela la perception de l'élite cléricale.

On remarque donc, durant cette période, un contraste significatif entre l'importance du cinéma dans les romans et celle qu'il détient socialement. Les romanciers en parlent peu; leurs personnages le fréquentent rarement et, s'ils en font mention, c'est la plupart du temps pour le dénigrer. Peut-on en déduire 
un certain asservissement des romanciers au discours officiel ou l'efficacité de la censure cléricale ou encore un écart entre le milieu intellectuel et le peuple?

Une étude des romans postérieurs à 1930 montre comment l'attitude des romanciers à l'égard du cinéma se transforme et comment le discrédit finira par s'estomper. À partir de la Deuxième Guerre mondiale, les romanciers se détacheront de l'idéologie dominante et qui plus est, la contesteront. En sourdine, la révolution tranquille se prépare et nous formulons l'hypothèse que le cinéma n'est pas étranger à l'instauration d'une nouvelle philosophie plus hédoniste, non plus axée sur les valeurs de sacrifice et de bonheur dans l'au-delà, mais sur l'épanouissement de l'individu dans cette vie. On reconnaîtra l'importance de l'imaginaire, et le cinéma — de même que le théâtre d'ailleurs - deviendra un élément important du développement de l'identité culturelle au même titre que le livre. La société canadienne-française se libérera de sa tradition passéiste pour devenir une nation ouverte sur l'avenir. Mais alors se définira une autre forme de méfiance envers l'image industrielle: parce que tout passe désormais par l'image, l'homme aura parfois l'impression d'être réduit à l'image qu'il projette.

Université Laurentienne

\section{NOTES}

1 Un article en témoigne: "Le cinématographe - Une des merveilles de notre siècle ", La Presse, lundi 29 juin 1896, p. 1.

2 Rappelons qu'en 1913, il y a déjà 70 salles de spectacles à Montréal. Joseph Gauvreau, inspecteur des théâtres et des scopes, estime à 125000 le nombre d'entrées par jour dans ces établissements, soit le quart de la population montréalaise. Voir à ce sujet Germain Lacasse, Histoires de scopes (Montréal: Cinémathèque québécoise I Musée du cinéma, 1988, p. 42).

3 Ce texte s'inspire de ma thèse de doctorat, La Présence du cinéma dans le roman canadien-français de 1896 à 1970 (Montpellier: Université Paul-Valéry -Montpellier III, 1994). Une copie de cette thèse a été déposée au centre de documentation de la Cinémathèque québécoise.

4 Cet échantillon est représentatif par son nombre, mais il l'est également par le nombre de romanciers et de romancières ainsi que par la proportion de romans choisie à chaque année. Dans les deux cas, nous avons toujours plus de $40 \%$ des romans publiés. De plus, notre corpus comprend toutes les œuvres majeures et un bon nombre d'œuvres mineures. 
5 Dans les romans du corpus, le mot "théâtre" est souvent utilisé pour désigner le cinéma; nous n'avons retenu que les occurrences où le mot "théâtre" désignait explicitement le cinéma.

6 Plusieurs auteurs se sont penchés sur des œuvres antérieures au cinéma afin d'en faire une "lecture cinématographique». Songeons à l'ouvrage de Paul L'Église, Une auvre de pré-cinéma. L'Énéide. Essai d'analyse filmique du premier chant (Paris: Les Nouvelles Éditions Debresse, 1958); et plus récemment, à celui d'Anne-Marie Baron, Balzac cinéaste (Paris: Klincksieck, 1990).

7 Ce chiffre n'inclut que les personnages identifiés clairement. Il exclut évidemment les occurrences où le romancier mentionne les foules, les groupes de jeunes, les amis, etc.

8 Notons que le déséquilibre de personnages masculins et féminins s'amenuise très vite. En effet de 1896 à 1919, on compte 10 hommes et 2 femmes seulement alors qu'au cours de la décennie suivante, les chiffres sont à peu près égaux : 5 hommes et 4 femmes. Pour les quatre décennies suivantes, les proportions demeurent à peu près équivalentes.

9 Ou du moins, comme le souligne Jeanne-Marie Clerc, que les romanciers aient voulu donner à penser qu'il était réservé presque exclusivement aux femmes. Voir à ce propos Jeanne-Marie Clerc, "Une culture infantile, féminine et populaire", Le Cinéma, témoin de l'imaginaire dans le roman français contemporain. Ecriture du visuel et transformations d'une culture (Berne: Peter Lang, 1984, p. 37-52).

10 Il s'agit du roman Robert Lozé (1903) de R.-E. Bouchette. La technique cinématographique $\mathrm{y}$ alimente une brève description: "Ce spectacle vaut à lui seul le voyage. Il se déroule avec des effets de cinématographe uniques et charmants " (Montréal: A.-P. Pigeon, 1903, p. 93). Second en liste, Damase Potvin dans Restons chez nous! (1908) retient aussi cette idée de mouvement lorsqu’il écrit que "[...] tout défile devant les regards fiévreux du mourant: les joies de famille, la jeunesse, les deuils, les morts, les amours, les voyages, tout comme dans un immense cinématographe [...]" (Québec: J.-Alfred Guay, 1908, p. 139).

11 L'histoire d'Ernest Ouimet, premier propriétaire d'une salle fixe de cinéma à Montréal, nous apprend qu'à l'occasion des élections municipales du $1^{\text {ct }}$ février 1904 et des élections fédérales et provinciales de novembre de la même année, la population fut invitée à se rendre à l'intersection des rues Sainte-Catherine et Hôtel-de-Ville. Ernest Ouimet avait fait étendre un grand drap blanc sur la façade de l'immeuble et il y projetait des films qui alternaient avec la projection des portraits des candidats élus. Pour plus de détails, voir Léon-H. Bélanger, Le Ouimetoscope: Léo-Ernest Ouimet et les débuts du cinéma québécois (Montréal : VLB éditeur, 1978, p. 31-32).

12 "Le plus grand crime dont on peut charger la plupart d'entre eux [les films], quels que soient leurs qualités ou leurs défauts, c'est de ruiner l'esprit de famille" (Anonyme, La Semaine religieuse de Québec, 22 novembre 1917, p. 461). Dans d'autres textes, on affirmera que la vie familiale est menacée par les mauvais exemples cinématographiques de divorces faciles, d'adultères et d'abandon du foyer pour partir à l'aventure.

13 Yves Lever, historien du cinéma, ira même jusqu'à dire: "[...] le principe du sacrifice, du devoir quotidien, de l'acceptation des limites ordinaires, en prend un coup devant ces images qui glorifient le plaisir et l'émergence de vies nouvelles" (L'Eglise et le cinéma au Québec (Thèse de maîtrise en théologie), Montréal: Université de Montréal, 1977, p. 77). 


\section{OUVRAGES CITÉS}

Aumont, Jacques et al. Esthétique du film. Paris: Nathan, 1983.

Baron, Anne-Marie. Balzac cinéaste. Paris: Klincksieck, 1990.

Baudry, Jean-Louis. L'Effet-cinéma. Paris: Albatros, 1978.

Bélanger, Léon-H. Le Ouimetoscope: Léo-Ernest Ouimet et les débuts du cinéma québécois. Montréal: VLB Éditeur, 1978.

Bernard, Harry. La Maison vide. Montréal : Bibliothèque de l'Action française, 1926.

Bernard, Harry. L'Homme tombé. Montréal: s.é., 1924.

Bernier, Hector. Ce que disait la flamme. Québec: Imprimerie de l'Événement, 1913.

Bouchette, Robert-Errol. Robert Lozé. Montréal : A.-P. Pigeon, 1903.

Clerc, Jeanne-Marie. Le Cinéma témoin de l'imaginaire dans le roman français contemporain. Écriture du visuel et transformations d'une culture. Berne: Peter Lang, 1984.

Deyglun, Henri. Les Aventuriers de l'amour. Montréal : Éditions Édouard Garand, 1929.

Goyette, Arsène. L'Ineffaçable Souillure. Sherbrooke: Imprimerie de La Tribune, 1926. Harvey, Jean-Charles. Marcel Faure. Montmagny: L'Imprimerie de Montmagny, 1922.

Hémon, Louis. Maria Chapdelaine. Montréal : Fides, 1970.

Jéhin, Jules. Les Aventures extraordinaires de deux Canayens. Montréal: Imprimerie A.-P. Pigeon, 1918.

Lacasse, Germain. Histoires de scopes. Montréal: Cinémathèque québécoise / Musée du cinéma, 1988.

Larivière, Jules-Ernest. L'Associée silencieuse. Montréal: Éditions Édouard Garand, 1925.

Larivière, Jules-Ernest. L'Tris bleu. Montréal : Éditions Édouard Garand, 1923.

L'Église, Paul. Une auvre de pré-cinéma. L'Énéïde. Essai d'analyse filmique du premier chant. Paris: Les Nouvelles Éditions Debresse, 1958.

Lever, Yves. L'Église et le cinéma au Québec (mémoire de maîtrise en théologie). Montréal : Université de Montréal, 1977.

Morin, Edgar. Les Stars. Paris: Galilée, 1982.

Mousseau, J.-M. Alfred. L'Envers du journalisme. Montréal : s.é., 1912.

Potvin, Damase. Le Membre. Québec: L'Événement, 1916.

Potvin, Damase. Restons chez nous! Québec: J.-Alfred Guay, 1908.

Tremblay, Micheline. La Présence du cinéma dans le roman canadien-français de 1896 à 1970 (thèse de doctorat). Montpellier: Université Paul-Valéry, 1994. 